\title{
Artificial Neural Network-Based System for PET Volume Segmentation
}

\author{
Mhd Saeed Sharif, ${ }^{1}$ Maysam Abbod, ${ }^{1}$ Abbes Amira, ${ }^{2}$ and Habib Zaidi ${ }^{3,4}$ \\ ${ }^{1}$ Department of Electronic and Computer Engineering, School of Engineering and Design, Brunel University, West London, \\ Uxbridge UB8 3PH, UK \\ ${ }^{2}$ Nanotechnology and Integrated Bioengineering Centre, University of Ulster, County Antrim BT37 0QB, UK \\ ${ }^{3}$ Division of Nuclear Medicine, Geneva University Hospital, 1211 Geneva, Switzerland \\ ${ }^{4}$ Geneva Neuroscience Center, Geneva University, 1211 Geneva, Switzerland
}

Correspondence should be addressed to Mhd Saeed Sharif, mhd.sharif@brunel.ac.uk

Received 14 May 2010; Revised 30 July 2010; Accepted 22 August 2010

Academic Editor: Yue Wang

Copyright (C) 2010 Mhd Saeed Sharif et al. This is an open access article distributed under the Creative Commons Attribution License, which permits unrestricted use, distribution, and reproduction in any medium, provided the original work is properly cited.

\begin{abstract}
Tumour detection, classification, and quantification in positron emission tomography (PET) imaging at early stage of disease are important issues for clinical diagnosis, assessment of response to treatment, and radiotherapy planning. Many techniques have been proposed for segmenting medical imaging data; however, some of the approaches have poor performance, large inaccuracy, and require substantial computation time for analysing large medical volumes. Artificial intelligence (AI) approaches can provide improved accuracy and save decent amount of time. Artificial neural networks (ANNs), as one of the best AI techniques, have the capability to classify and quantify precisely lesions and model the clinical evaluation for a specific problem. This paper presents a novel application of ANNs in the wavelet domain for PET volume segmentation. ANN performance evaluation using different training algorithms in both spatial and wavelet domains with a different number of neurons in the hidden layer is also presented. The best number of neurons in the hidden layer is determined according to the experimental results, which is also stated Levenberg-Marquardt backpropagation training algorithm as the best training approach for the proposed application. The proposed intelligent system results are compared with those obtained using conventional techniques including thresholding and clustering based approaches. Experimental and Monte Carlo simulated PET phantom data sets and clinical PET volumes of nonsmall cell lung cancer patients were utilised to validate the proposed algorithm which has demonstrated promising results.
\end{abstract}

\section{Introduction}

Medical images can be obtained using various modalities such as positron emission tomography (PET), singlephoton emission computed tomography (SPECT), computed tomography (CT), magnetic resonance imaging (MRI), and ultrasound (US). PET is a molecular imaging technique used to probe physiological functions at the molecular level rather than to look at anatomy through the use of trace elements such as carbon, oxygen, and nitrogen which have a high abundance within the human body. PET plays a central role in the management of oncological patients beside the other main components such as diagnosis, staging, treatment, prognosis, and followup. Owing to its high sensitivity and specificity, PET is effective in targeting specific functional or metabolic signatures that may be associated with various diseases. Among all diagnostic and therapeutic procedures, PET is unique in the sense that it is based on molecular and pathophysiological mechanisms and employs radioactively labeled biological molecules as tracers to study the pathophysiology of the tumour in vivo to direct treatment and assess response to therapy. The leading current area of clinical use of PET is in oncology, where ${ }^{18} \mathrm{~F}$-fluorodeoxyglucose (FDG) remains the most widely used tracer. FDG-PET has already had a large valuable effect on cancer staging and treatment, and its use in clinical oncology practice continues to evolve [1-5].

The main challenge of PET is its low spatial resolution which results in the so-called partial volume effect. This effect should be reduced to the minimum level, so that the required 
information can be accurately quantified and extracted from the analysed volume. On the other hand, the increasing number of patient scans beside the widespread application of PET have raised the urgent need for effective volume analysis techniques to aid clinicians in clinical diagnosis and set the proper plan for treatment. Analysing and extracting the proper information from PET volumes can be performed by deploying image segmentation and classification approaches which provide richer information than that obtained directly from qualitative assessment alone performed on the original PET volumes [6]. The need for accurate and fast analysis for medical volume segmentation leads to exploit artificial intelligence (AI) techniques. These include artificial neural networks (ANN), expert systems, robotics, genetic algorithms, intelligent agents, logic programming, fuzzy logic, neurofuzzy, natural language processing, and automatic speech recognition $[7,8]$.

ANN is one of the powerful AI techniques that has the capability to learn from a set of data and construct weight matrices to represent the learning patterns. ANN has great success in many applications including pattern classification, decision making, forecasting, and adaptive control. Many research studies have been carried out in the medical field utilising ANN for medical image segmentation and classification with different medical imaging modalities. Multilayer perceptron (MLP) neural network (NN) have been used by [9] to identify breast nodule malignancy using sonographic images. A multiple classifier system using five NNs and five sets of texture features extraction for the characterization of hepatic tissue from CT images is presented in [10]. Kohonen self-organizing NN for segmentation and a multilayer backpropagation NN for classification for multispectral MRI images have been used in [11]. Kohonen NN was also used for image segmentation in [12]. Computer-aided diagnostic (CAD) scheme to detect lung nodules using a multiresolution massive training artificial neural network (MTANN) is presented in [13].

The aim of this paper is to develop a robust, efficient PET volume segmentation system using ANN. The proposed system is evaluated using different training algorithms and its performance assessed using different metrics. ANN outputs are also compared with the outputs of conventional approaches including thresholding and clustering using experimental PET phantom studies and clinical volumes of nonsmall cell lung cancer patients.

This paper is organised as follows. Section 2 presents mathematical background for the selected approaches. The materials and methods used are described in Section 3. Experimental results and their discussion are given in section 4, and finally some conclusions are presented in Section 5.

\section{Mathematical Background}

2.1. Mathematical Model of a Neuron. ANN is a mathematical model which emulates the activity of biological neural networks in the human brain. It consists of two or several layers each one has many interconnected group of neurons. Each neuron in the ANN has a number of inputs (the input vector $P$ ) and one output $(Y)$. The input vector elements are multiplied by weights $w_{1,1}, w_{1,2}, \ldots, w_{1, R}$, and the weighted values are fed to the summing junction. Their sum is simply the dot product (W.P) of the single-row matrix $W$ and the vector $P$. The neuron has a bias $b$, which is summed with the weighted inputs to form the net input $n$. This sum, $n$, is the argument of the transfer function $f$ [14]

$$
\begin{gathered}
n=\sum_{i=1}^{R} w_{1, i} \cdot p_{i}+b, \\
Y=f(W \cdot P+b), \\
Y(j)=f\left[\sum_{i=1}^{R} w_{1, i}(j) \cdot p_{i}(j)+b\right] .
\end{gathered}
$$

The learning process can be summarized in the following steps: (1) the initial weights are randomly assigned, (2) the neuron is activated by applying inputs vector and desired output $\left(Y_{d}\right)$, and (3) calculation of the actual output $(Y)$ at iteration $j=1$ as illustrated in (3), where iteration $j$ refers to the $j$ th training example presented to the neuron. The following step is to update the weights to obtain the output consistent with the training examples, as illustrated in

$$
w_{1, i}(j+1)=w_{1, i}(j)+\Delta w_{1, i}(j)
$$

where $\Delta w_{1, i}(j)$ is the weight correction at iteration $j$. The weight correction is computed by using the delta rule in

$$
\Delta w_{1, i}(j)=\alpha * p_{i}(j) * e(j)
$$

where $\alpha$ is the learning rate and $e(j)$ is the error which can be given by

$$
e(j)=Y_{d}(j)-Y(j)
$$

Finally, the iteration $j$ is increased by one, and the previous two steps are repeated until the convergence is reached.

\subsection{Thresholding}

2.2.1. Hard Thresholding. Thresholding is the simplest precursory technique for image segmentation. This methodology attempts to determine an intensity value that can separate the slice $g(x, y)$ into two parts [15]. All voxels with intensities $f(x, y)$ larger than the threshold value $T$ are allocated into one class, and all the others into another class.

$$
g(x, y)= \begin{cases}f(x, y) & \text { if } f(x, y)>T \\ 0 & \text { if } f(x, y)<T\end{cases}
$$

Thresholding approach does not consider the spatial characteristics of a volume; it is sensitive to noise and intensities variation. Thresholding approach has been used extensively in the literature as ground truth to compare some of the proposed schemes for medical image segmentation $[16,17]$. 
2.2.2. Soft Thresholding. Soft thresholding is more complex process compared to hard thresholding. This approach replaces each voxel which has a greater value than the threshold value by the difference between the threshold and the current voxel values. Soft thresholding could put into evidence some important regions as the region of interest (ROI) in this study.

2.2.3. Adaptive Thresholding. Otsu's method has been used as a third approach, which chooses the threshold that minimizes the intraclass variance of the black and white voxels in the volume [18]. Likewise, other variants of adaptive thresholding based on source-to-background ratio were also reported [4].

2.3. Multiresolution Analysis. Multiresolution analysis (MRA) is designed to give good time resolution and poor frequency resolution at high frequencies, and poor time resolution and good frequency resolution at low frequencies. It enables the exploitation of slice characteristics associated with a particular resolution level, which may not be detected using other analysis techniques [19-21]. The wavelet transform for a function $f(t)$ can be defined as follows:

$$
X_{\psi(a, b)}=\int_{-\infty}^{\infty} f(t) \psi_{(a, b)}(t) d t
$$

where

$$
\psi_{(a, b)}(t)=\frac{1}{\sqrt{a}} \psi\left(\frac{t-b}{a}\right)
$$

The parameters $a, b$ are called the scaling and shifting parameters, respectively $[20,22]$. Haar wavelet filter will be used in the experimental study at different levels of decomposition. The Haar wavelet transform (HWT) of a twodimensional slice can be performed using two approaches: the first one is called standard decomposition of a slice, where the one-dimensional HWT is applied to each row of voxel values followed by another one-dimensional HWT on the column of the processed slice. The other approach is called nonstandard decomposition, which alternates between the one-dimensional HWT operations on rows and columns. HWT serves as a prototype for all other wavelet transforms. Like all wavelet transforms, HWT decomposes a slice into four subimages of half the original size. HWT is conceptually simple, fast, memory efficient, and can be reversed without the edge effects that are associated with other wavelet transforms. HWT is a matrix-vector-based operation and can be formulated as follows:

$$
\begin{gathered}
H=\frac{1}{2}\left(\begin{array}{cc}
1 & 1 \\
1 & -1
\end{array}\right), \\
O=I \times H, \\
O=\left((I H)^{T} H\right)^{T}=H^{T} I H, \\
I=\left(H^{-1}\right)^{T} O H^{-1},
\end{gathered}
$$

where $I$ is $2 \times 2$ input matrix, $H$ contains the Haar coefficients, and $O$ is the output matrix. Equations (12) and (13) show the transposed and reconstructed matrices, respectively. MRA has been used in the literature for different applications [22-24].

2.4. Clustering. Clustering techniques aim to classify each voxel in a volume into the proper cluster, then these clusters are mapped to display the segmented volume. The most commonly used clustering technique is the $K$ means method, which clusters $n$ voxels into $K$ clusters ( $K$ less than $n$ ) [25]. This algorithm chooses the number of clusters, $K$, then randomly generates $K$ clusters and determines the cluster centers. The next step is assigning each point in the volume to the nearest cluster center, and finally recompute the new cluster centers. The two previous steps are repeated until the minimum variance criterion is achieved. This approach is similar to the expectationmaximization algorithm for Gaussian mixture in which they both attempt to find the centers of clusters in the volume. Its main objective is to achieve a minimum intracluster variance V

$$
V=\sum_{i=1}^{K} \sum_{x_{j} \in S_{i}}\left(x_{j}-\mu_{i}\right)^{2},
$$

where $K$ is the number of clusters, $S=1,2, \ldots, K$, and $\mu_{i}$ is the mean of all voxels in the cluster $i$. $K$-means approach has been used with other techniques for clustering medical images [26].

\section{Materials and Methods}

3.1. The Proposed System. The proposed medical volume segmentation system is illustrated in Figure 1 . The $3 \mathrm{D}$ PET volume acquired from the scanner goes through the preprocessing block, which enhances the quality of slice features and removes most of the noise from each slice. The enhanced volume can be processed using three approaches, the first processing block is thresholding which removes the background and unnecessary information producing a volume consists of two classes the background and the ROI. The second approach is $K$-means clustering technique which classifies each slice in PET volume into an appropriate number of clusters. The third approach is ANN which is used in both spatial and wavelet domains. The preprocessed PET volume is fed first to the ANN which is trained to detect the tumour. In another block, the PET volume is transformed into the wavelet domain using HWT at different levels of decomposition. This transform decomposes the volume and produces the approximation, horizontal, vertical, and diagonal features for each slice. The approximation features are fed to another ANN for classifying and quantifying the tumour. The outputs of ANNs are compared in the next step with the outputs of the other two approaches, while the best outputs are selected and displayed. The system has been tested using experimental and simulated phantom studies and clinical oncological PET volumes of nonsmall cell lung cancer patients. 


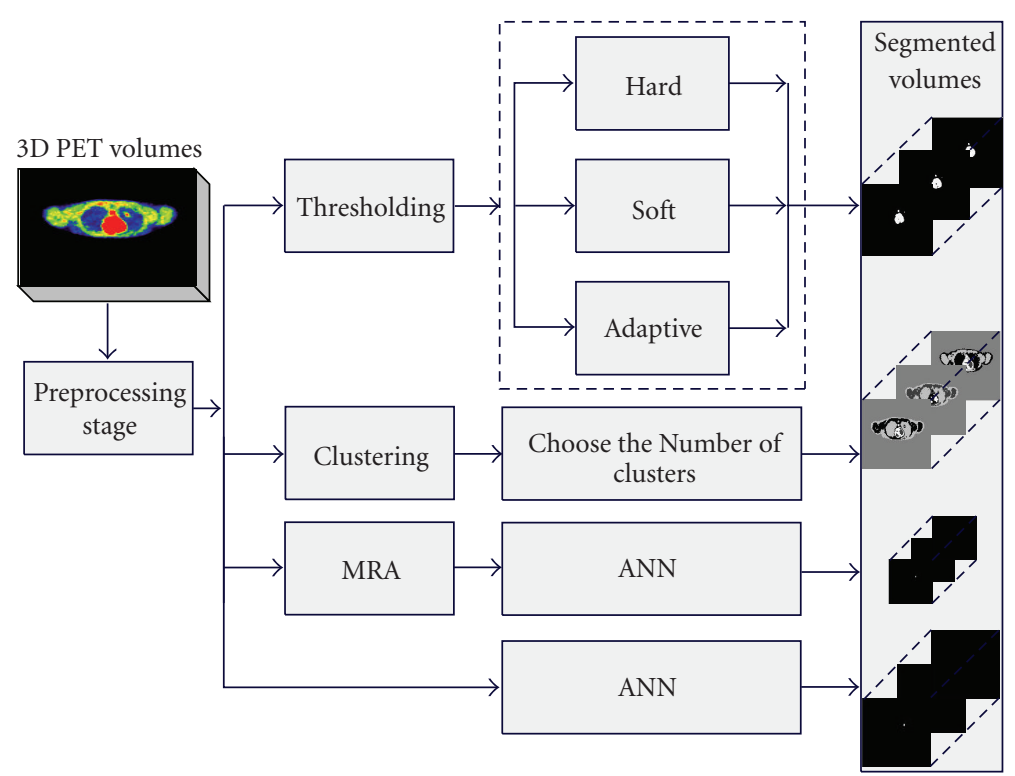

Figure 1: Proposed system for PET volume segmentation.

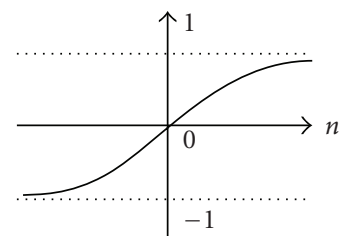

(a)

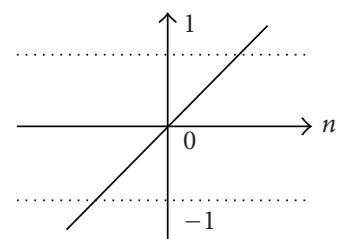

(b)
Figure 2: Activation functions: (a) Tangent-sigmoid function, (b) Linear function.

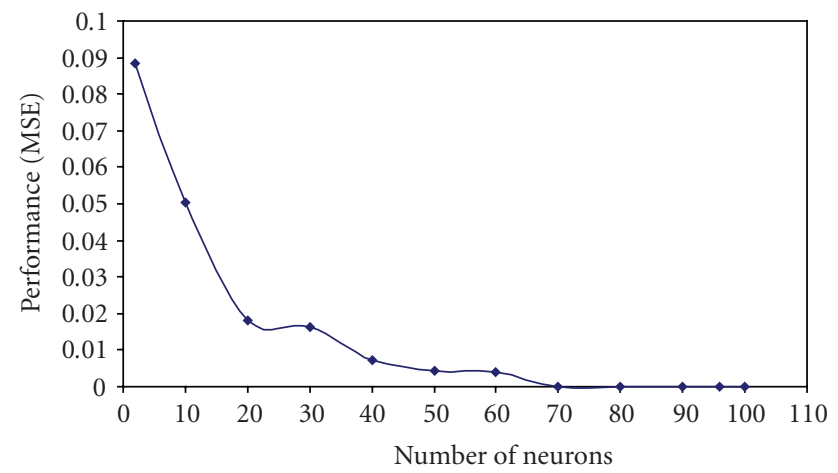

FIgURE 3: Evaluation of the number of neurons in the first hidden layer with neural network performance using MSE.

3.2. Phantom Studies. In this study, PET volumes containing simulated tumour have been utilised. Two phantom data sets have been used. The first data set is obtained using NEMA IEC image quality body phantom which consists of an elliptical water-filled cavity with six spherical inserts suspended by plastic rods of volumes $0.5,1.2,2.6,5.6,11.5$, and $26.5 \mathrm{ml}$ (inner diameters of $10,13,17,22,28$, and $37 \mathrm{~mm}$ ). The voxel size is $4.07 \mathrm{~mm} \times 4.07 \mathrm{~mm} \times 5 \mathrm{~mm}$, while the size

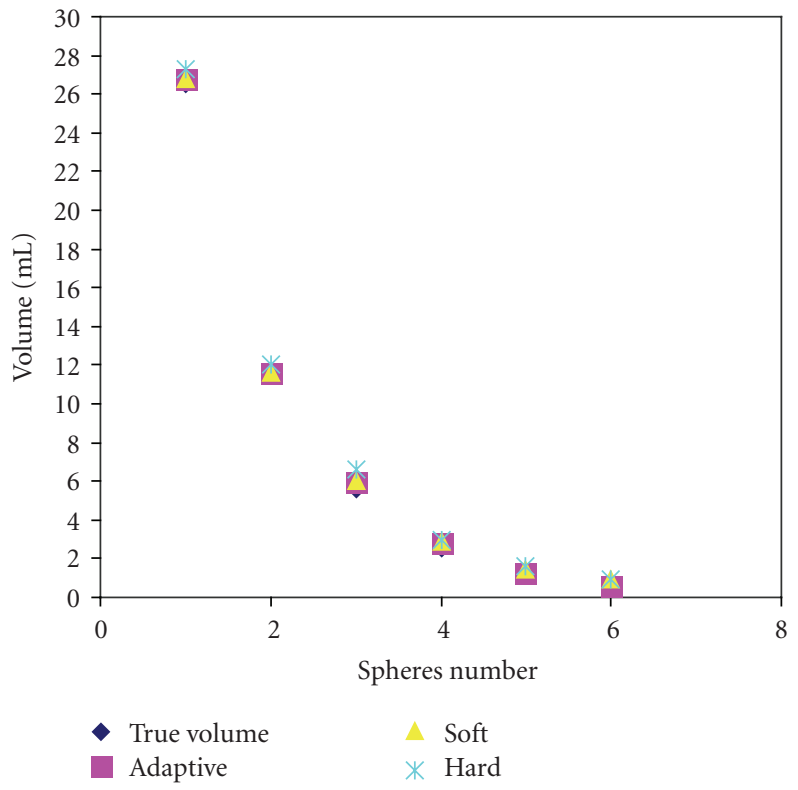

FIGURE 4: The segmented volume for all spheres in data set 1 using adaptive, soft, and hard thresholding approaches, respectively.

of the obtained phantom volume is $168 \times 168 \times 66$. This phantom was extensively used in the literature for assessment of image quality and validation of quantitative procedures [27-30]. Other variants of multisphere phantoms have also been suggested [31]. The PET scanner used for acquiring the data is the Biograph 16 PET/CT scanner (Siemens Medical Solution, Erlangen, Germany) operating in 3D mode [32]. Following Fourier rebinning and model-based scatter correction, PET images were reconstructed using two-dimensional iterative normalized attenuation-weighted ordered subsets expectation maximization (NAW-OSEM). 


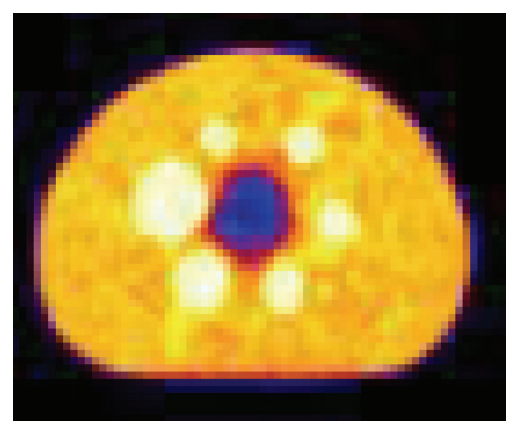

(a)

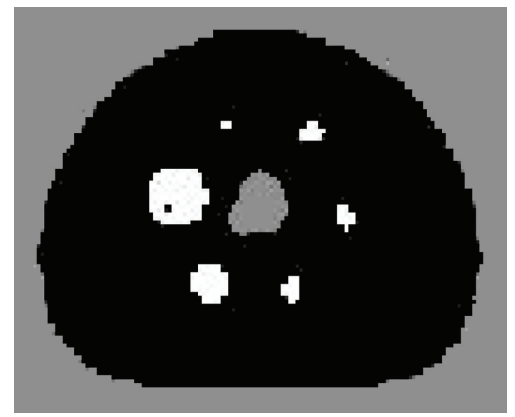

(c)

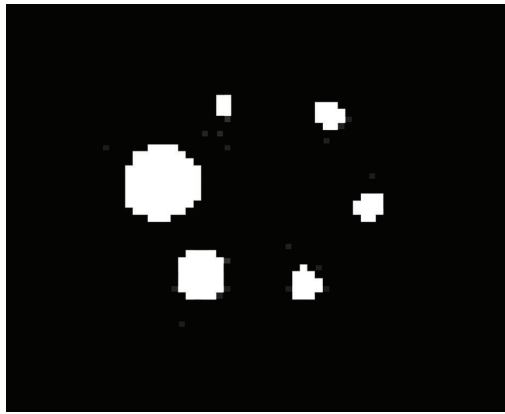

(b)

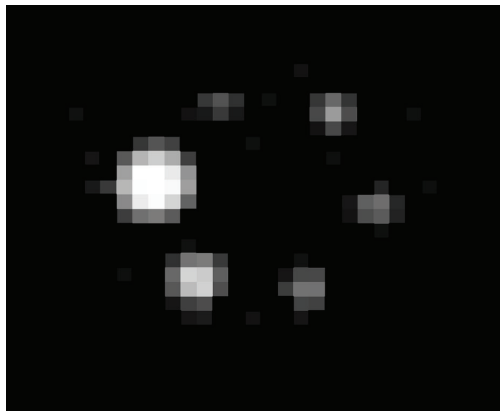

(d)

FIgure 5: Phantom data set 1: (a) Original PET image $(168 \times 168)$, (b) thresholded image $(168 \times 168)$, (C) clustered image $(168 \times 168)$, (d) segmented image $(84 \times 84)$ using ANN and MRA, zoomed by a factor of 2 .

TABLE 1: Tumours characteristics for the second data set.

\begin{tabular}{lcccc}
\hline Tumour & \multicolumn{2}{c}{ Isotropic } & \multicolumn{2}{c}{ Nonisotropic } \\
Number & Position & Size & Position & Size \\
\hline 1 & slice 68 & 2 voxels & slice 142 & 2 voxels \\
2 & slice 57 & 3 voxels & slice 119 & 3 voxels \\
3 & slice 74 & 2 voxels & slice 155 & 2 voxels \\
\hline
\end{tabular}

CT-based attenuation correction was used to reconstruct the PET emission data. The default parameters used were ordered OSEM iterative reconstruction with four iterations and eight subsets followed by a postprocessing Gaussian filter (kernel full-width half-maximal height, $5 \mathrm{~mm}$ ).

The second data set consists of Monte Carlo simulations of the Zubal antropommorphic model where two volumes were generated [33]. The first volume contains a matrix with isotropic voxels, the size of this volume is $128 \times 128 \times 180$. The second volume contains the same matrix of the first one but with nonisotropic voxels having a matrix size of $128 \times$ $128 \times 375$. The voxel size in both volumes is $5.0625 \mathrm{~mm}$ $\times 5.0625 \mathrm{~mm} \times 2.4250 \mathrm{~mm}$. The second data volume has 3 tumours in the lungs whose characteristics are given in Table 1 .

3.3. Clinical PET Studies. Clinical PET volumes of patients with histologically proven NSCLC (clinical Stage Ib-IIIb) who have undertaken a diagnostic whole-body PET/CT scan were used for assessment of the proposed segmentation technique [34]. Patients fasted no less than 6 hours before
PET/CT scanning. The standard protocol involved intravenous injection of ${ }^{18} \mathrm{~F}$-FDG followed by a physiologic saline $(10 \mathrm{ml})$. The injected FDG activity was adjusted according to patient's weight using the following formula: A (Mbq) = weight $(\mathrm{Kg}) 4+20$. After $45 \mathrm{~min}$ uptake time, freebreathing PET and CT images were acquired. The data were reconstructed using the same procedure described for the phantom studies. The maximal tumour diameters measured from the macroscopic examination of the surgical specimen served as ground truth for comparison with the maximum diameter estimated by the proposed segmentation technique. The voxel size is $5.31 \mathrm{~mm} \times 5.31 \mathrm{~mm} \times 5 \mathrm{~mm}$, while the size of the obtained clinical volume is $128 \times 128 \times 178$.

\section{Results and Discussion}

4.1. NEMA Image Quality Phantom. An experimental study has been run at the beginning to determine the best ANN design and algorithms. Multilayer feedforward NNs [8] consists of input layer (144 neurons), hidden layer (variant number of hidden neurons), and outputs layer (1) has been chosen first to determine the best number of hidden neurons. To evaluate the effect of the number of neurons in the hidden layer and achieve the best ANN performance for our application, different numbers of neurons in the hidden layer have been used. The maximum number of iterations used in the ANN is 1000. The experiment has been repeated 10 times for each chosen number of the hidden neurons, and the average was considered for that number. Hyperbolic tangent sigmoid transfer function has been used for all layers 


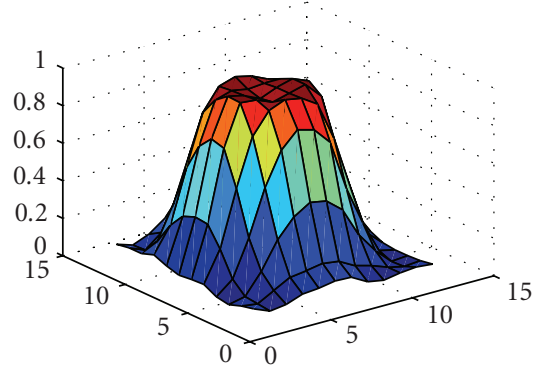

(a)

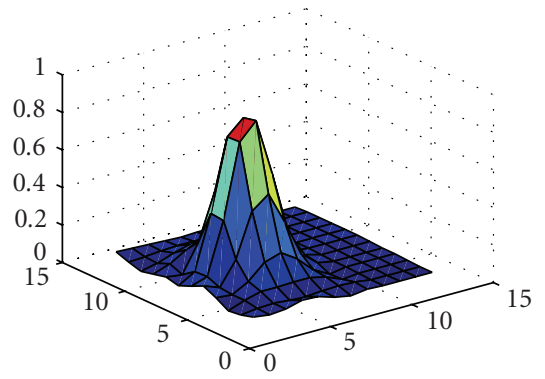

(d)

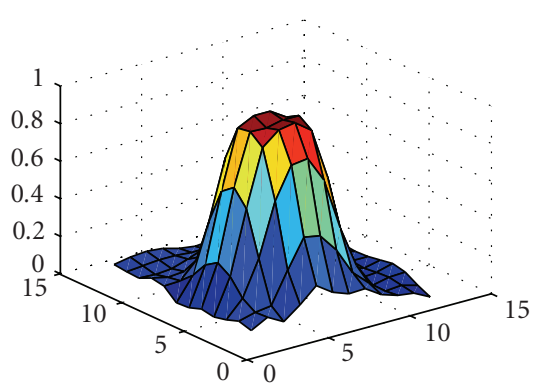

(b)

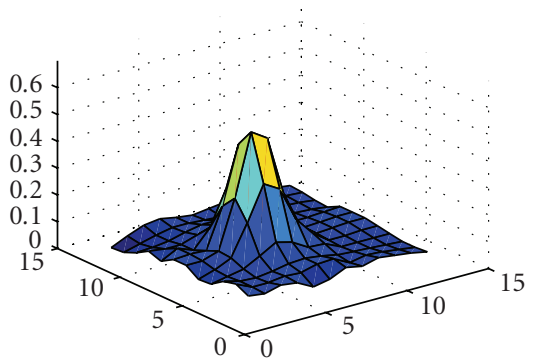

(e)

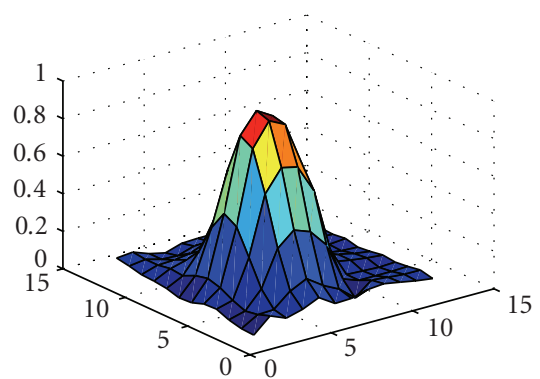

(c)

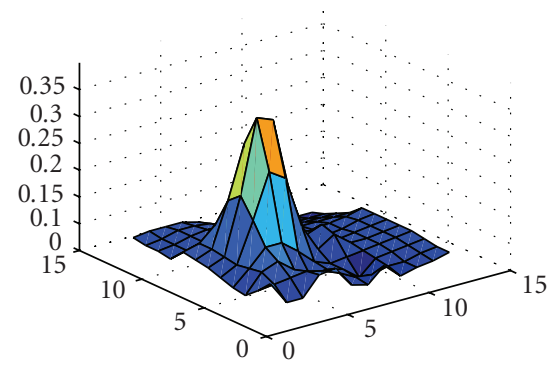

(f)

FIGURE 6: Segmented sphere surface plot for phantom data set 1. Voxel values scaled in [0..1] on Z axis, and voxels number is within [0..12] on $\mathrm{X}$ and $\mathrm{Y}$ axes: (a) sphere 1, (b) sphere 2, (c) sphere 3, (d) sphere 4, (e) sphere 5, and (f) sphere 6.

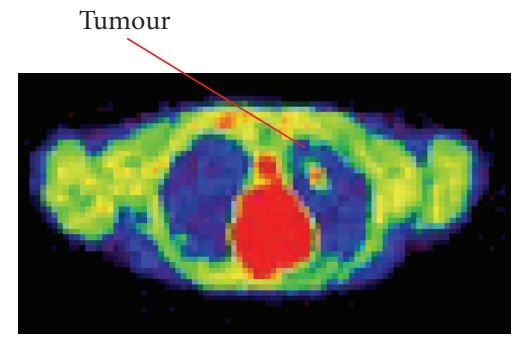

(a)

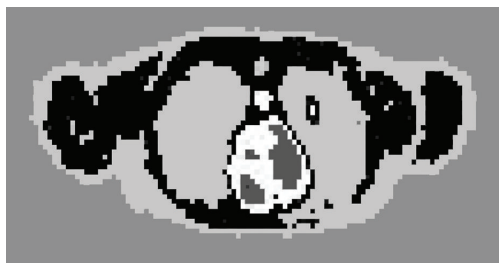

(c)

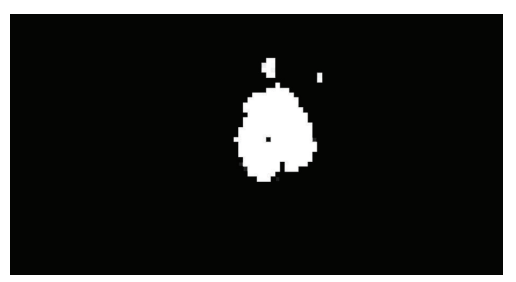

(b)

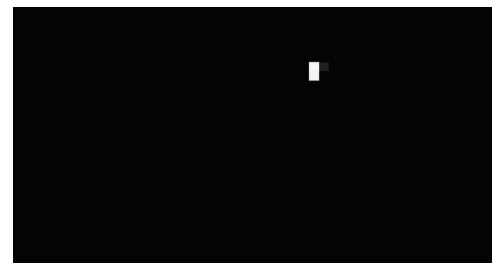

(d)

FIgure 7: Phantom data set 2. (Tumour 1): (a) Original PET image $(128 \times 128)$, (b) thresholded image $(128 \times 128)$, (C) clustered image $(128 \times 128)$, (d) segmented image $(64 \times 64)$ using ANN and MRA, zoomed by a factor of 2 , where tumour $1(2$ voxel $)$ is detected.

except the output layer where the linear activation function is used. The two activation functions are illustrated in Figure 2. Levenberg-Marquardt backpropagation training algorithm has been used during the evaluation of neurons numbers in the hidden layer [35] to validate the best design for the ANN, which is suitable for the proposed application. Figure 3 presents the number of neurons in the first hidden layer with the performance measured using mean-squared error (MSE) at 1000 iterations. The results obtained after this evaluation shows that the best number of the hidden neurons which corresponds to the smallest MSE, and good ANN outputs is 70 hidden neurons.

Using the achieved ANN structure, different training algorithms have been evaluated in the next step to achieve the best ANN performance. In this evaluation the same ANN structure, sufficient training cases and 1000 epochs have been considered. The following training algorithms have been used in this part of the study. BFGS quasi-Newton backpropagation [36], bayesian regulation backpropagation (BR) [37], conjugate gradient backpropagation with Powell-Beale 


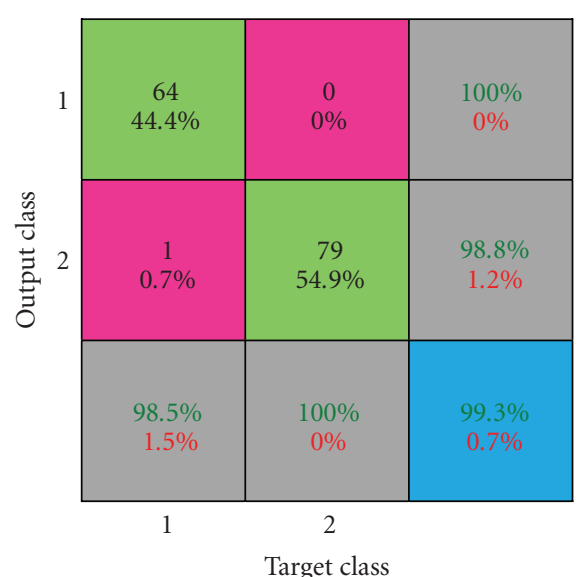

(a)

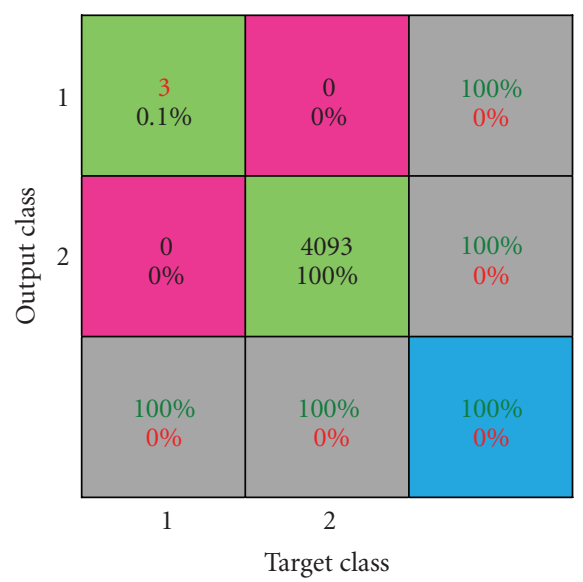

(c)

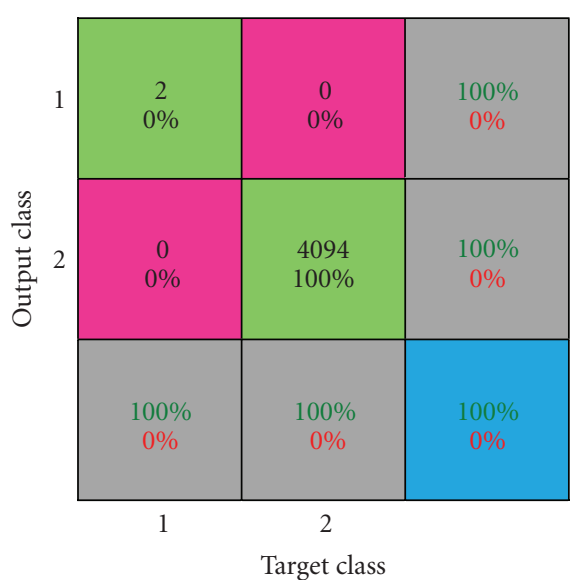

(b)

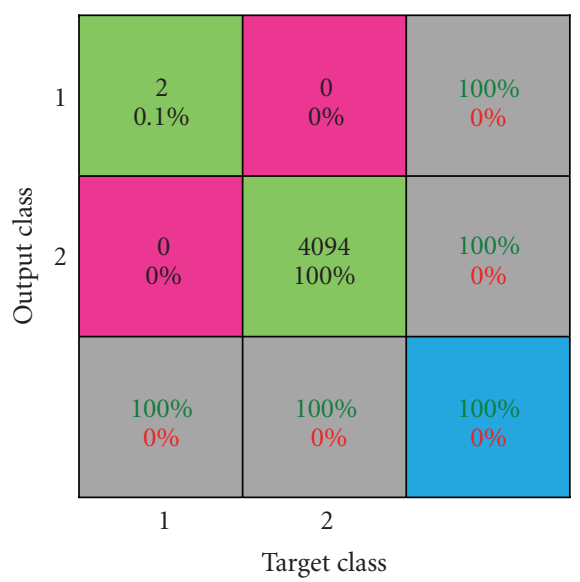

(d)

Figure 8: Confusion matrix: (a) for phantom data set 1, sphere 1, (b) phantom data set 2, tumour 1, (c) phantom data set 2, tumour 2, and (d) for phantom data set 2 , tumour 3 .

restarts (CGB) [38], conjugate gradient backpropagation with Fletcher-Reeves updates (CGF) [39], conjugate gradient backpropagation with Polak-Ribire updates (CGP) [39], gradient descent backpropagation (GD) [40], gradient descent with momentum backpropagation (GDM) [41], gradient descent with adaptive learning rate backpropagation (GDA) [42], gradient descent with momentum and adaptive learning rate backpropagation (GDX) [43], Levenberg-Marquardt backpropagation (LM) [35], one-step secant backpropagation (OSS) [44], random order incremental training with learning functions (R), resilient backpropagation (RP) [45], and scaled conjugate gradient backpropagation (SCG) [46]. The average of the performance and the required time for each of these training algorithms are presented in Table 2, this experiment has been repeated for 10 times and the standard deviation for the performance achieved is 4.15E07. The best outputs associated with the best performance was achieved using Levenberg-Marquardt backpropagation training algorithm. This algorithm is using a combination of techniques which allows the $\mathrm{NN}$ to be trained efficiently. This combination includes backpropagation, gradient descent approach, and Gauss-Newton technique [47, 48].
After determining the main design parameters of ANN, a feedforward ANN with one hidden layer (70 hidden neurons), one outputs layer (1) has been used in the study of PET data sets. The training algorithm used with this network is Levenberg-Marquardt backpropagation algorithm. In this application, $70 \%$ of the first data set have been used for training (46 slices), $15 \%$ for validating (10 slices), and $15 \%$ for testing (10 slices). A window of $12 \times 12$ voxels has been used to scan each input slice. The size of this window is chosen to include all the spheres even the biggest one. The utilisation of this window has reduced the input features size fed into the ANN each time without losing the slice details in addition to reduce the required computational time. The input features of the ANN have been extracted in spatial and wavelet domains. For both domains an ANN with 144 inputs, 70 hidden neurons, and (1) outputs layer has been used. The input features in the spatial domain are the voxels of each processed slice. While the utilised wavelet filter decomposes each slice from the input volume and produces four types of coefficients. The approximation coefficients produced by the HWT represent the most detailed information about the analysed slice. The size of these coefficients $(84 \times 84)$ is half 


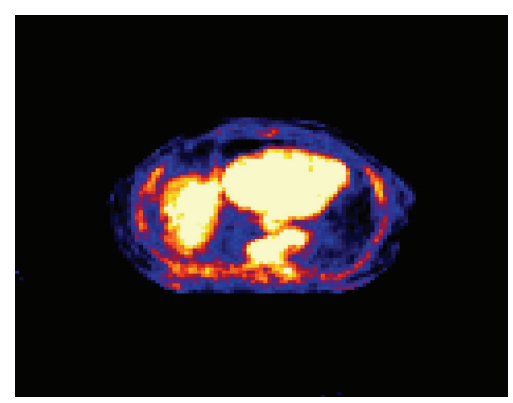

(a)

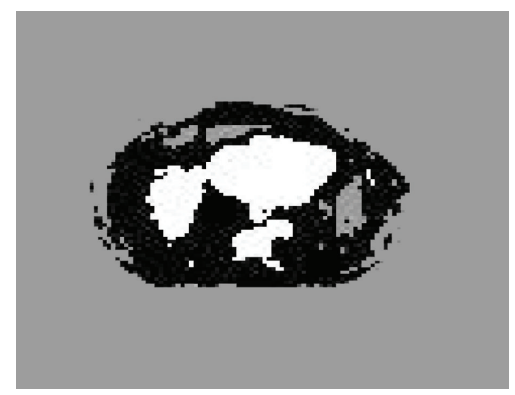

(c)

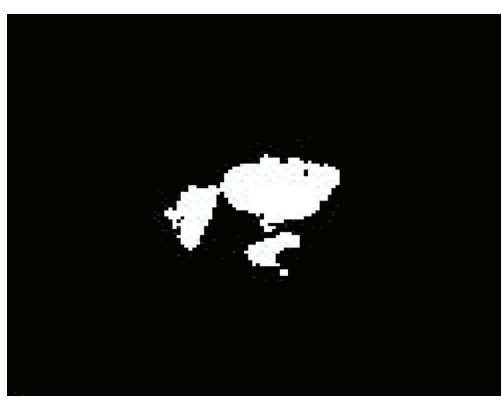

(b)

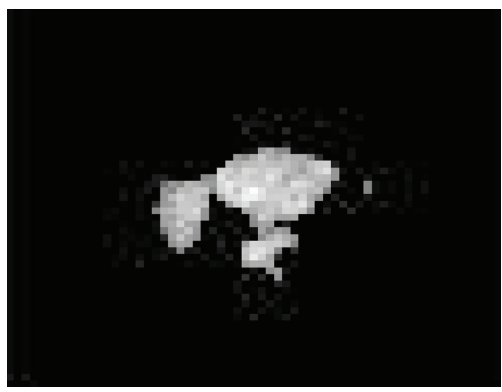

(d)

FIGURE 9: Illustration of algorithm performance on clinical PET data showing: (a) original PET image $(128 \times 128)$, (b) thresholded image $(128 \times 128),(C)$ clustered image $(128 \times 128)$, and $(d)$ segmented image $(64 \times 64)$ using ANN and MRA, zoomed by a factor of 2 .

TABLE 2: Evaluation of the effect of different training algorithms on the performance of multilayer feedforward NN with 144-70-1 topology.

\begin{tabular}{lcc}
\hline Training function & Time $(\mathrm{sec})$ & Performance (MSE) \\
\hline BFG & 58 & 0.000426 \\
BR & 35 & $3.69 \mathrm{e}-6$ \\
CGB & 11 & 0.0128 \\
CGF & 11 & 0.0156 \\
CGP & 13 & 0.0160 \\
GD & 6 & 0.117 \\
GDM & 6 & 0.100 \\
GDA & 6 & 0.0950 \\
GDX & 6 & 0.0383 \\
LM & 27 & $1.32 \mathrm{E}-7$ \\
OSS & 13 & 0.0198 \\
R & 226 & 0.0503 \\
RP & 6 & 0.0183 \\
SCG & 10 & 0.0154 \\
\hline
\end{tabular}

of the original size. The ANN achieved good performance with very small MSE, 2.39E-16.

An objective evaluation of the artificial intelligence system (AIS) outputs has been performed by comparing the sphere computed volume $(\mathrm{CV})$ with its true original volume (TV). The experimental results have been repeated 10 times, and the average of the sphere volume measured using ANN is calculated. The standard deviation for the volume of sphere 1 is 0.0971 , for sphere 2 is 0.1170 , for sphere 3 is 0.1185 , for sphere 4 is 0.1232 , for sphere 5 is 0.1258 , and for sphere 6 is 0.1293 . The CV obtained from the ANN, and the percentage of the absolute relative error (ARE \%) for each sphere are presented in Table 3. ANN has clearly detected all spheres, where spheres 1, 2 , and 3 are accurately segmented whereas spheres 4 is overestimated, and sphere 5 and 6 are underestimated. It is worth mentioning that the proposed system has shown better performance compared to the thresholding and clustering based approaches which are used as ground truth. Adaptive, soft, and hard thresholding approaches have been also used to perform the segmentation. The best results obtained from these approaches is by using adaptive threshold method which is used for the comparison with the other assessed techniques. Figure 4 presents the obtained spheres volumes using three thresholding approaches. Table 3 illustrates a comparison between the assessed approaches in term of ARE percentage. Thresholding approach has overestimated the volume of all spheres, while the exploitation of $K$ means clustering approach underestimates the volume of all spheres, particularly spheres 5 and 6 .

The segmented slices from thresholding, clustering and ANN in the wavelet domain are illustrated in Figure 5, where Figure $5(\mathrm{~d})$ is zoomed for illustration purpose. The three-dimensional shaded surface for each segmented sphere obtained from ANN are plotted in Figure 6, where the voxel values are scaled in [0..1] on $\mathrm{Z}$ axis and voxels number is within $[0 . .12]$ on the remaining two axes.

4.2. Simulated Zubal Phantom. The proposed segmentation system was able to detect tumours in the second phantom data set with isotropic voxels. The first tumour with size 
TABLE 3: Comparison of sphere volumes and ARE between TV and CV for different segmentation algorithms assessed including thresholding, clustering and ANN.

\begin{tabular}{lccccccc}
\hline & \multicolumn{2}{c}{ Spheres } & \multicolumn{2}{c}{ Thresholding } & \multicolumn{2}{c}{ Clustering } & \multicolumn{2}{c}{ ANN } \\
No. & TV $(\mathrm{ml})$ & CV $(\mathrm{ml})$ & ARE $\%$ & CV $(\mathrm{ml})$ & ARE $\%$ & CV $(\mathrm{ml})$ & ARE \% \\
\hline 1 & 26.52 & 26.74 & 0.81 & 25.73 & 2.97 & 26.54 & 0.08 \\
2 & 11.49 & 11.62 & 1.07 & 11.01 & 4.22 & 11.51 & 0.10 \\
3 & 5.58 & 5.96 & 6.97 & 5.28 & 5.35 & 5.63 & 0.95 \\
4 & 2.57 & 2.81 & 9.08 & 2.40 & 6.89 & 2.63 & 2.13 \\
5 & 1.15 & 1.29 & 11.98 & 1.03 & 10.03 & 1.10 & 4.54 \\
6 & 0.52 & 0.61 & 15.76 & 0.46 & 11.52 & 0.49 & 5.88 \\
\hline
\end{tabular}

of 2 voxels was clearly detected in slice 68 . Figure 7 shows the segmented slices from thresholding, clustering, and ANN in the wavelet domain for this tumour. The second and third tumours with size 3 and 2 voxels, respectively, were also clearly detected in slice 57 and 74, respectively. Similar results have been achieved for detecting tumours in the second data set with nonisotropic voxels. On the other hand similar segmented slices have been obtained using ANN in the spatial domain, however, more computational time is required for processing all data sets in this domain.

4.3. Performance Evaluation. In the field of AI a number of performance metrics can be employed to evaluate the performance of ANN. A confusion matrix is a visualisation tool typically used in supervised and unsupervised learning approaches. Each row of the matrix represents the instances in a predicted class, while each column represents the instances in an actual class. One benefit of a confusion matrix is that it is easy to see if the system is confusing two classes (the tumour and the remaining tissues). The confusion matrix for the first data set shows that 1 voxel out of 65 ones in the first segmented sphere was misclassified, Figure 8(a). Where the percent in the green box refers to each class prediction accuracy. While the percent in the pink box refers to the misclassified voxels accuracy in each class. The gray boxes represent the percents of classified voxels numbers in each class in green, and the percent of the error in each class in red. The blue box represents the total percent of all classes in green and the total error in these classes in red. All the numbers in the confusion matrix are represented as a percentage.

The confusion matrix for the second data set, tumour 1 is illustrated in Figure 8(b). The two voxels of this tumour were precisely classified in one class and the remaining voxels (4094) classified in the other class. The confusion matrix for the second data set, tumour 2, shows that the 3 voxels were precisely classified as a first class and the remaining voxels (4093) classified in the other class. The obtained result is presented in Figure 8(c), while Figure 8(d) illustrates the confusion matrix for the second data set, tumour 3 . The two voxels of tumour 3 were precisely classified as a first class and the remaining voxels (4094) classified in the other class.

The other performance checking approach is receiver operating characteristic (ROC). This approach can be represented by plotting the fraction of true positives rate (TPR) versus the fraction of false positives rate (FPR), where the perfect point in the ROC curve is the point $(0,1)$. The ROC curve for the first data set is located near the perfect point and the FPR for the sphere voxels number is near the 0 point. Perfect ROC has been obtained for the second data set and the FPR for tumour voxels number is 0 .

4.4. Clinical PET Studies. The proposed approaches have been also tested on clinical PET volumes of nonsmall cell lung cancer patients. A subjective evaluation based on the clinical knowledge has been carried out for the output of the proposed approaches. The tumour in these slices has a maximum diameter on the $y$-axis of $90 \mathrm{~mm}$ (estimated by histology). The segmented tumour using ANN in spatial domain and wavelet domain (after scaling) has a diameter of $90.1 \mathrm{~mm}$. The segmented volumes using the proposed approaches outlines a well defined contour as illustrated in Figure 9.

\section{Conclusions}

An artificial intelligence system based on multilayer artificial neural networks was proposed for PET volume segmentation. Different training algorithms have been utilised in this study to validate the best algorithm for the targeted application. Two PET phantom data sets and a clinical PET volume of nonsmall cell lung cancer patient have been used to evaluate the performance of the proposed system. Objective and subjective evaluation for the system outputs have been carried out. Confusion matrix and receiver operating characteristic were also used to judge the performance of the trained neural network. Experimental and simulated phantom results have shown a good performance for the ANN in detecting the tumours in spatial and wavelet domains for both phantom and clinical PET volumes. Accurate tumour quantification was also achieved through this system. Ongoing research is focusing on further validation of the proposed algorithm in a clinical setting and the exploitation of other artificial intelligence tools and feature extraction techniques.

\section{Acknowledgment}

This paper was supported by the Swiss National Science Foundation under Grant no. 3152A0-102143. 


\section{References}

[1] D. Mankoff, M. Muzi, and H. Zaidi, "Quantitative analysis in nuclear oncologic imaging," in Quantitative Analysis in Nuclear Medicine Imaging, H. Zaidi, Ed., pp. 494-536, Springer, New York, NY, USA, 2006.

[2] D. W. G. Montgomery, A. Amira, and H. Zaidi, "Fully automated segmentation of oncological PET volumes using a combined multiscale and statistical model," Medical Physics, vol. 34, no. 2, pp. 722-736, 2007.

[3] M. Aristophanous, B. C. Penney, and C. A. Pelizzari, "The development and testing of a digital PET phantom for the evaluation of tumor volume segmentation techniques," Medical Physics, vol. 35, no. 7, pp. 3331-3342, 2008.

[4] H. Vees, S. Senthamizhchelvan, R. Miralbell, D. C. Weber, O. Ratib, and H. Zaidi, "Assessment of various strategies for $18 \mathrm{~F}-$ FET PET-guided delineation of target volumes in high-grade glioma patients," European Journal of Nuclear Medicine and Molecular Imaging, vol. 36, no. 2, pp. 182-193, 2009.

[5] S. Basu, "Selecting the optimal image segmentation strategy in the era of multitracer multimodality imaging: a critical step for image-guided radiation therapy," European Journal of Nuclear Medicine and Molecular Imaging, vol. 36, no. 2, pp. 180-181, 2009.

[6] H. Zaidi and I. El Naqa, "PET-guided delineation of radiation therapy treatment volumes: a survey of image segmentation techniques," European Journal of Nuclear Medicine and Molecular Imaging, vol. 37, pp. 1-37, 2010.

[7] G. Dreyfus, Neural Networks Methodology and Applications, Springer, Berlin, Germany, 2005.

[8] M. A. Arbib, The Handbook of Brain Theory and Neural Networks, Massachusetts Institute of Technology, Cambridge, Mass, USA, 2003.

[9] S. Joo, W. K. Moon, and H. C. Kim, "Computer-aidied diagnosis of solid breast nodules on ultrasound with digital image processing and artificial neural network," in Proceedings of the 26th Annual International Conference of the IEEE Engineering in Medicine and Biology Society (EMBS '04), pp. 1397-1400, 2004.

[10] S. G. Mougiakakou, I. Valavanis, K. S. Nikita, A. Nikita, and D. Kelekis, "Characterization of CT liver lesions based on texture features and a multiple neural network classification scheme," in Proceedings of the 25th Annual International Conference of the IEEE Engineering in Medicine and Biology Society (EMBS '03), vol. 2, pp. 1287-1290, 2003.

[11] W. E. Reddick, J. O. Glass, E. N. Cook, T. D. Elkin, and R. J. Deaton, "Automated segmentation and classification of multispectral magnetic resonance images of brain using artificial neural networks," IEEE Transactions on Medical Imaging, vol. 16, no. 6, pp. 911-918, 1997.

[12] C. C. Reyes-Aldasoro and A. Aldeco, "Image segmentation and compression using neural networks," in Advances in Artificial Perception and Robotics CIMAT, 2000.

[13] K. Suzuki, H. Abe, H. MacMahon, and K. Doi, "Imageprocessing technique for suppressing ribs in chest radiographs by means of massive training artificial neural network (MTANN)," IEEE Transactions on Medical Imaging, vol. 25, no. 4, pp. 406-416, 2006.

[14] G. F. Luger, Artificial Intelligence: Structures and Strategies for Complex Problem Solving, Pearson Education Inc., 2009.

[15] P. K. Sahoo, S. Soltani, and A. K. C. Wong, "A survey of thresholding techniques," Computer Vision, Graphics and Image Processing, vol. 41, no. 2, pp. 233-260, 1988.
[16] A. Kanakatte, J. Gubbi, N. Mani, T. Kron, and D. Binns, "A pilot study of automatic lung tumor segmentation from positron emission tomography images using standard uptake values," in Proceedings of the IEEE Symposium on Computational Intelligence in Image and Signal Processing (CIISP '07), pp. 363-368, 2007.

[17] M. Sezgin and B. Sankur, "Survey over image thresholding techniques and quantitative performance evaluation," Journal of Electronic Imaging, vol. 13, no. 1, pp. 146-168, 2004.

[18] N. Otsu, "A threshold selection method from gray-level histograms," IEEE Transactions on Systems, Man, and Cybernetics, vol. 9, no. 1, pp. 62-66, 1979.

[19] S. G. Mallat, "A theory for multiresolution signal decomposition: the wavelet representation," IEEE Transactions on Pattern Analysis and Machine Intelligence, vol. 11, no. 7, pp. 674-693, 1989.

[20] R. Gonzalez and R. Woods, Digital Image Processing, PrenticeHall, Upper Saddle River, NJ, USA, 2001.

[21] A. Amira, S. Chandrasekaran, D. W. G. Montgomery, and I. Servan Uzun, "A segmentation concept for positron emission tomography imaging using multiresolution analysis," Neurocomputing, vol. 71, no. 10-12, pp. 1954-1965, 2008.

[22] K. M. Rajpoot and N. M. Rajpoot, "Hyperspectral colon tissue cell classification," in Medical Imaging, Proceedings of SPIE, 2004.

[23] G. Fan and X.-G. Xia, "Wavelet-based texture analysis and synthesis using hidden Markov models," IEEE Transactions on Circuits and Systems I, vol. 50, no. 1, pp. 106-120, 2003.

[24] H. Liu, Z. Chen, X. Chen, and Y. Chen, "Multiresolution medical image segmentation based on wavelet transform," in Proceedings of the 27th Annual International Conference of the IEEE Engineering in Medicine and Biology Society (EMBS '05), pp. 3418-3421, September 2005.

[25] A. K. Jain and R. C. Dubes, Algorithms for Clustering Data, Prentice-Hall, Upper Saddle River, NJ, USA, 1988.

[26] H. P. Ng, S. H. Ong, K. W. C. Foong, P. S. Goh, and W. L. Nowinski, "Medical image segmentation using k-means clustering and improved watershed algorithm," in Proceedings of the 7th IEEE Southwest Symposium on Image Analysis and Interpretation, pp. 61-65, March 2006.

[27] C. Jonsson, R. Odh, P.-O. Schnell, and S. A. Larsson, "A comparison of the imaging properties of a 3- and 4-ring biograph PET scanner using a novel extended NEMA phantom," in Proceedings of the IEEE Nuclear Science Symposium and Medical Imaging Conference (NSS-MIC '07), vol. 4, pp. 28652867, November 2007.

[28] M. D. R. Thomas, D. L. Bailey, and L. Livieratos, "A dual modality approach to quantitative quality control in emission tomography," Physics in Medicine and Biology, vol. 50, no. 15, pp. N187-N194, 2005.

[29] H. Bergmann, G. Dobrozemsky, G. Minear, R. Nicoletti, and M. Samal, "An inter-laboratory comparison study of image quality of PET scanners using the NEMA NU 2-2001 procedure for assessment of image quality," Physics in Medicine and Biology, vol. 50, no. 10, pp. 2193-2207, 2005.

[30] H. Herzog, L. Tellmann, C. Hocke, U. Pietrzyk, M. E. Casey, and T. Kawert, "NEMA NU2-2001 guided performance evaluation of four siemens ECAT PET scanners," IEEE Transactions on Nuclear Science, vol. 51, no. 5, pp. 2662-2669, 2004.

[31] J. M. Wilson and T. G. Turkington, "Multisphere phantom and analysis algorithm for PET image quality assessment," Physics in Medicine and Biology, vol. 53, no. 12, pp. 3267-3278, 2008.

[32] H. Zaidi, F. Schoenahl, and O. Ratib, "Geneva PET/CT facility: design considerations and performance characteristics of two 
commercial (Biograph 16/64) scanners," European Journal of Nuclear Medicine and Molecular Imaging, vol. 34, supplement 2, p. S166, 2007.

[33] S. Tomeï, A. Reilhac, D. Visvikis et al., "OncoPET-DB: a freely distributed database of realistic simulated whole body $18 \mathrm{~F}$ FDG PET images for oncology," IEEE Transactions on Nuclear Science, vol. 57, no. 1, pp. 246-255, 2010.

[34] A. van Baardwijk, G. Bosmans, L. Boersma et al., "PETCT-based auto-contouring in non-small-cell lung cancer correlates with pathology and reduces interobserver variability in the delineation of the primary tumor and involved nodal volumes," International Journal of Radiation Oncology, Biology, Physics, vol. 68, no. 3, pp. 771-778, 2007.

[35] B. G. Kermani, S. S. Schiffman, and H. T. Nagle, "Performance of the Levenberg-Marquardt neural network training method in electronic nose applications," Sensors and Actuators B, vol. 110, no. 1, pp. 13-22, 2005.

[36] P. E. Gill, W. Murray, and M. H. Wright, Practical Optimization, Academic Press, New York, NY, USA, 1981.

[37] D. MacKay, "Bayesian interpolation," Neural Computation, vol. 4, no. 3, pp. 415-447, 1992.

[38] M. J. D. Powell, "Restart procedures for the conjugate gradient method," Mathematical Programming, vol. 12, no. 1, pp. 241254, 1977.

[39] L. E. Scales, Introduction to Non-Linear Optimization, Springer, Berlin, Germany, 1985.

[40] Y. Bengio, P. Simard, and P. Frasconi, "Learning longterm dependencies with gradient descent is difficult," IEEE Transactions on Neural Networks, vol. 5, no. 2, pp. 157-166, 1994.

[41] A. Bhaya and E. Kaszkurewicz, "Steepest descent with momentum for quadratic functions is a version of the conjugate gradient method," Neural Networks, vol. 17, no. 1, pp. 65-71, 2004.

[42] S. Iranmanesh, "A differential adaptive learning rate method for back-propagation neural networks," in Proceedings of the 10th WSEAS International Conference on Neural Networks, 2009.

[43] G. D. Magoulas, M. N. Vrahatis, and G. S. Androulakis, "Improving the convergence of the backpropagation algorithm using learning rate adaptation methods," Neural Computation, vol. 11, no. 7, pp. 1769-1796, 1999.

[44] R. Battiti, "First and second order methods for learning: between steepest descent and Newton's method," Neural Computation, vol. 4, no. 2, pp. 141-166, 1992.

[45] M. Riedmiller and H. Braun, "A direct adaptive method for faster backpropagation learning: the RPROP algorithm," in Proceedings of the IEEE International Conference on Neural Networks (ICNN '93), pp. 586-591, April 1993.

[46] M. F. Moller, "A scaled conjugate gradient algorithm for fast supervised learning," Neural Networks, vol. 6, no. 4, pp. 525$533,1993$.

[47] X. Yu, M. O. Efe, and O. Kaynak, "A backpropagation learning framework for feedforward neural networks," in Proceedings of the IEEE International Symposium on Circuits and Systems (ISCAS '01), vol. 3, pp. 700-702, May 2001.

[48] T. L. Fine, Feedforward Network Methodology, Springer, Berlin, Germany, 1999. 

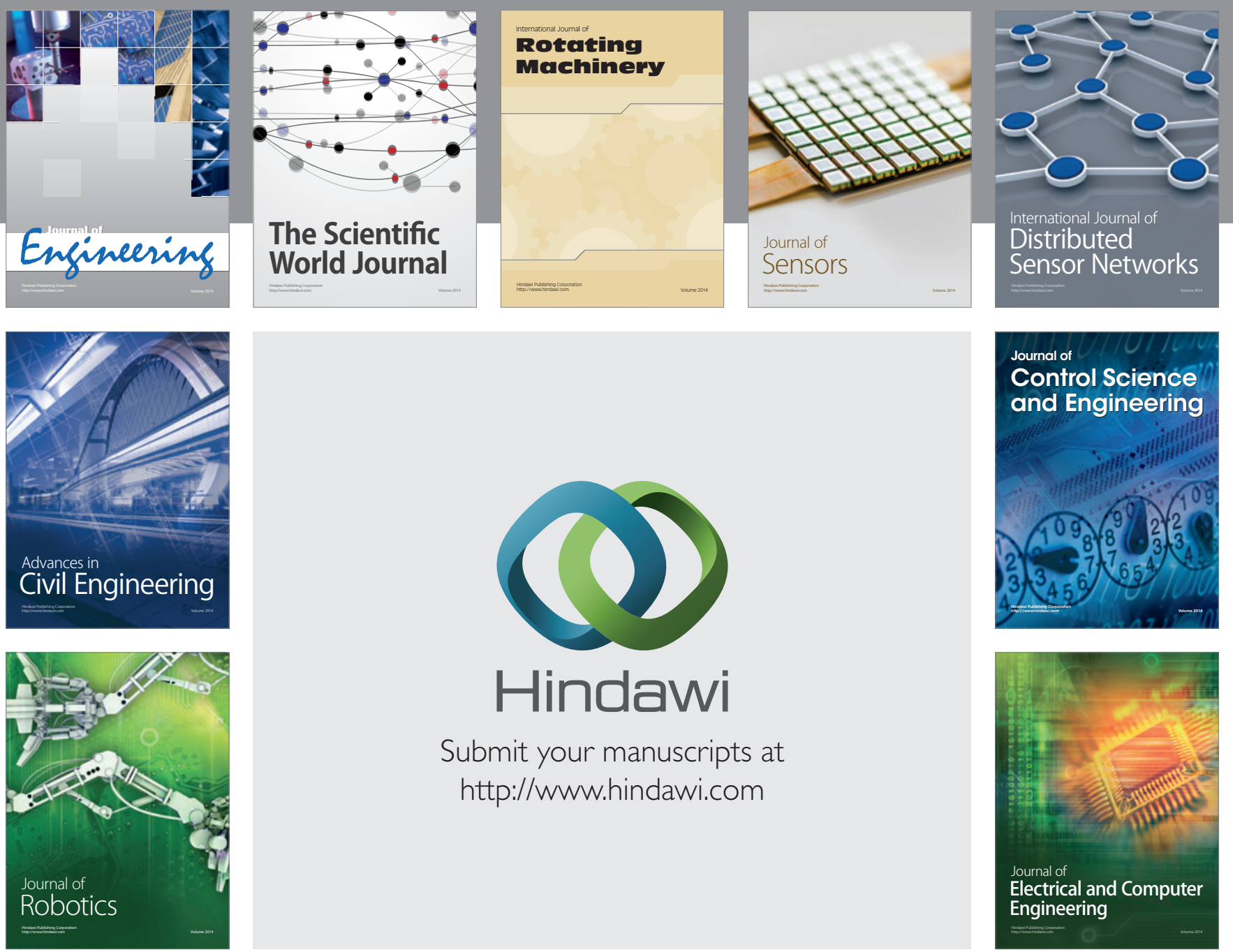

Submit your manuscripts at

http://www.hindawi.com
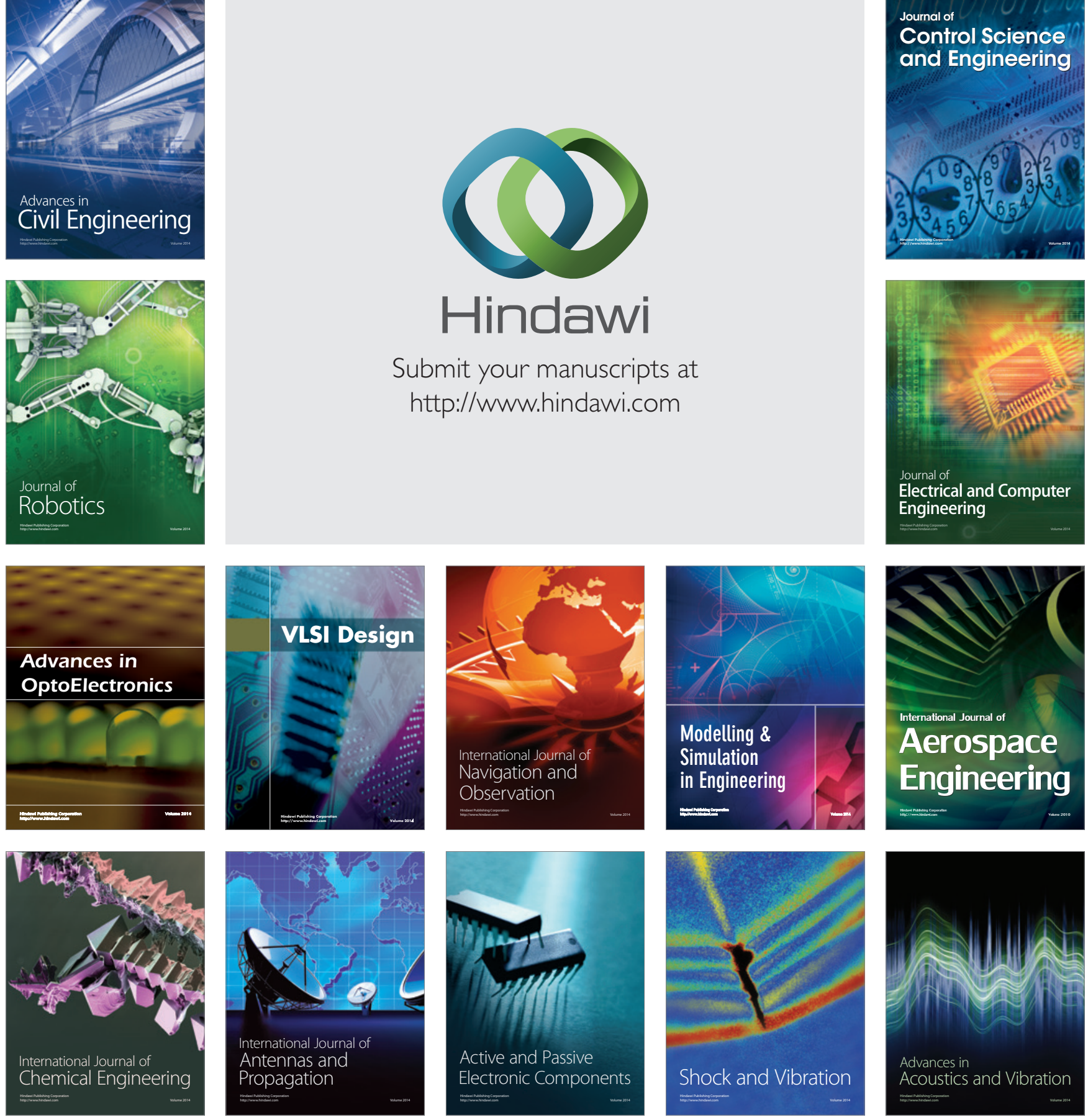